\title{
Minimally invasive cardiac surgery in a patient with Klippel-Feil syndrome: A case report
}

\author{
Rajesh Kishan Rao ${ }^{1,}{ }^{*}$, Girish Basappa ${ }^{1}$, Jaya prakash HM ${ }^{1}$, Jeeva Vijayan ${ }^{1}$ and Nagaraj DM ${ }^{2}$ \\ ${ }^{1}$ Department of Cardiothoracic Surgery, Sri Jayadeva Institute of Cardiovascular Sciences \& Research (SJICVS\&R), \\ Bangalore, Karnataka, India \\ ${ }^{2}$ Department of Cardiac Anaesthesia, Sri Jayadeva Institute of Cardiovascular Sciences \& Research (SJICVS\&R), Bangalore, Karnataka, India
}

\begin{abstract}
Klippel-Feil syndrome is a rare congenital disorder characterized by short neck, restricted neck movements and low posterior hair line. We present a case of Klippel- Feil syndrome in a 9-year-old girl, who also had left Sprengel's deformity, bilateral overcrowding of upper ribs, and atrial septal defect. Atrial septal defect was repaired by minimally invasive technique (Right vertical infra axillary thoracotomy).
\end{abstract}

Keywords: Klippel - Feil syndrome; right vertical infra axillary thoracotomy; atrial septal defect

*Corresponding author: Dr. Rajesh Kishan Rao, Department of
Cardiothoracic Surgery; Sri Jayadeva Institute of Cardiovascular
Sciences \& Research (SJICVS\&R), Bannerghatta Road, Jayanagar
$9^{\text {th }}$ block, Bangalore-560069, Karnataka, India. Tel.: +91
9886964031; Fax: 080-26534477; Email: rajesh.mk5207@gmail.
com

Received 02 November 2018; Revised 05 December 2018; Accepted 12 December 2018; Published 18 December 2018

Citation: Rao RK, Basappa G, Prakash HMJ, Vijayan J, Nagaraj DM. Minimally invasive cardiac surgery in a patient with Klippel-Feil syndrome: A case report. J Med Sci Res. 2019; 7(1):18-20. DOI: http://dx.doi.org/10.17727/JMSR.2019/7-5

Copyright: (C) 2019 Rao RK et al. Published by KIMS Foundation and Research Center. This is an open-access article distributed under the terms of the Creative Commons Attribution License, which permits unrestricted use, distribution, and reproduction in any medium, provided the original author and source are credited.

\section{Introduction}

Klippel-Feil syndrome (KFS) was first described in 1912 by Maurice Klippel and Andre Feil. It results from faulty segmentation of long axis of the developing embryo during $2^{\text {nd }}$ to $8^{\text {th }}$ week of gestation. Apart from classical triad it is associated with other skeletal, visceral and cardiac anomalies. Most common cardiac anomalies include ventricular septal defects, atrial septal defects [1, 2] and coarctation of aorta.

\section{Case report}

A 9-year-old girl was referred for surgical closure of atrial septal defect (ASD), as it was not suitable for device closure. On physical examination child had features of KFS, short neck, restricted neck movements and low posterior hair line (Figure 1a). Child also had kyphosis, torticollis, left Sprengel's deformity i.e., congenital short and high scapula (Figure 1b). There was no neurological abnormality. X-ray of the neck (lateral view) showed extensive fusion of the cervical vertebra (Figure 2a). Plain 
radiography of the chest revealed bilateral overcrowding of upper ribs with fusion of upper thoracic vertebra, bilateral absence of lower ribs, cardiomegaly with pulmonary plethora (Figure $2 b$ ). Abdominal ultrasonography revealed no anomalies.

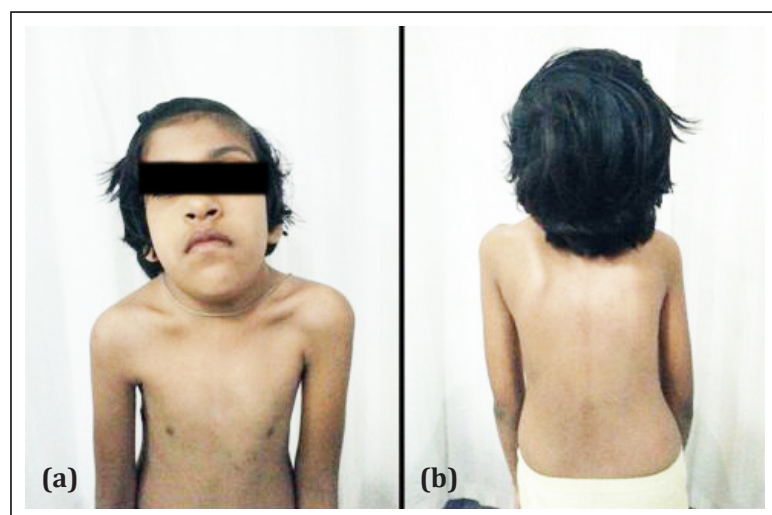

Figure 1: (a) A 9-year-old girl with Klippel-Feil syndrome, (b) Left Sprengel's deformity and low posterior hairline.

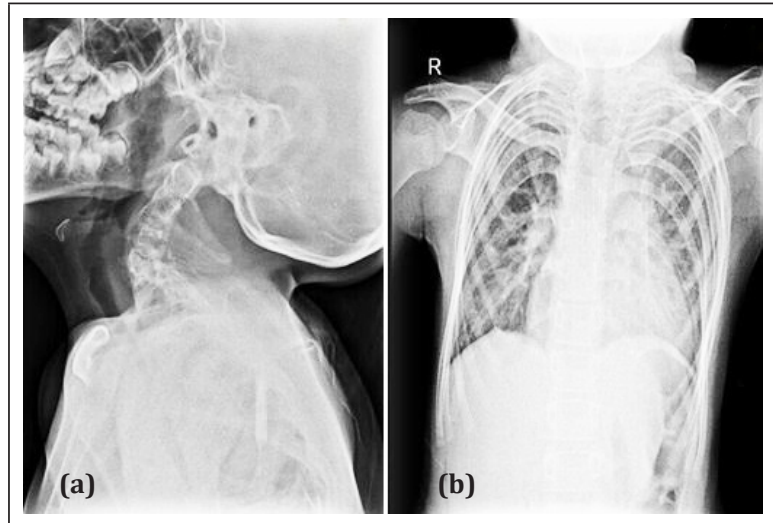

Figure 2: (a) X-ray cervical spine (lateral view ), extensive fusion of cervical vertebra, (b) X-ray chest (AP view): Bilateral overcrowding of upper ribs, fusion of upper thoracic vertebra, bilateral absence of lower ribs, cardiomegaly with pulmonary plethora.

After informed consent, child was taken up for surgery. Endotracheal intubation was done with conventional laryngoscope uneventfully. Child was placed in left lateral decubitus position with $60^{\circ}$ to $70^{\circ}$ and right upper limb was brought over the head and supported by a sling. A $3 \mathrm{~cm}$ vertical incision was made in the mid axillary line. Right pleural cavity was entered through $4^{\text {th }}$ intercostal space. After pericardiotomy, cardio pulmonary bypass was established by cannulating, ascending aorta, superior vena cava and right femoral vein. ASD was closed with autologous pericardial patch under cardioplegic arrest. Intra-operative and postoperative period was uneventful. She was discharged on $4^{\text {th }}$ post-operative day. At the time of discharge she was in sinus rhythm with no residual defects with healed axillary thoracotomy wound (Figure 3).

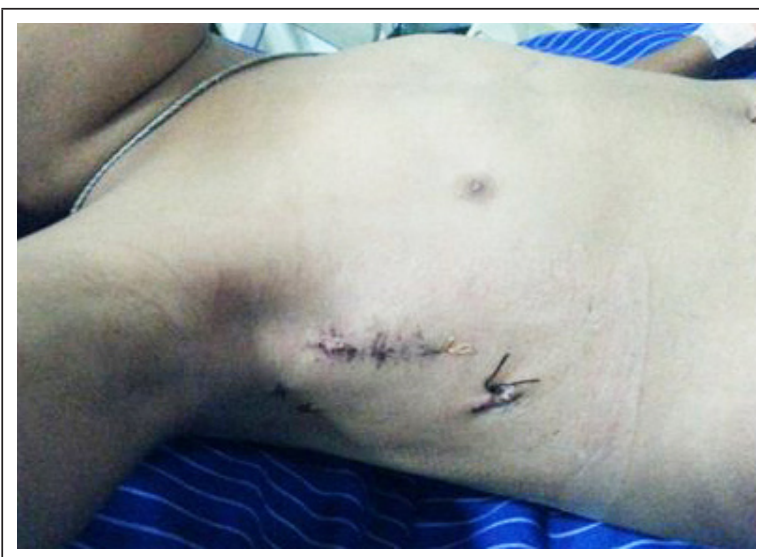

Figure 3: Healed scar in right mid axillary line.

\section{Discussion}

KFS is a rare congenital anomaly characterized by a triad of short neck, restricted neck movements and low posterior hair line. It is associated with other skeletal, cardiac and genitourinary anomalies. In this case the child had ASD [1,2], bilateral overcrowding of upper ribs, kyphosis, left Sprengel's deformity [3], torticollis, fusion of upper thoracic vertebra and bilateral absent lower ribs.

As the girl was in prepubescent age group we approached through right vertical infra axillary thoracotomy (RVIAT). This approach does not interfere with future breast development [4], and also has excellent cosmetic results.

Main technical challenge in patients with KFS is the airway management during general anaesthesia (due to unstable cervical spine and abnormal atlantooccipital junction) which has to be performed without neck extension [5]. Endotracheal intubation was done with conventional laryngoscope uneventfully. Another challenge was entry into the right pleural cavity and avoid excessive rib spreading, as there was overcrowding of upper ribs. In RVIAT we usually do central cannulation which not only requires longer incision and also excessive rib spreading. In this case we wanted to minimize rib spreading as much as possible, which can be achieved by reducing the number of cannulae in the operative field, i.e., by peripheral arterial and venous cannulation. However we avoid peripheral arterial cannulation in children, 
in view of small size femoral artery and future risk of femoral artery stenosis. We planned for peripheral venous cannulation (cannulation of right internal jugular vein and right femoral vein). However right internal jugular vein cannulation was difficult as the child had a short neck. So we cannulated ascending aorta, superior vena cava and right femoral vein.

Few cases of KFS with ASD which were repaired by median sternotomy have been reported $[1,2]$. To the best of our knowledge this is the first case of KFS, where ASD was repaired through minimally invasive approach (RVIAT). Also we wanted to highlight few technical challenges in this case.

\section{Conclusion}

To conclude, repair of simple congenital cardiac defects can be safely performed through RVIAT, in patients with KFS. However proper preoperative evaluation, planning by surgical, anaesthetic and perfusion team is essential.

\section{Conflicts of interest}

Authors declare no conflicts of interest.

\section{References}

[1] Karaagac AT, Yildirim AI. A type 3 Klippel-Feil syndrome case with atrial septal defect and atrial septal aneurysm. Int J Pediat Health Care Adv. 2016; 3(1):13-16.

[2] Bejiqui R, Retkoceri R, Bejiqui H, Zeka N, Maloku A, et al. Klippel-Feil syndrome associated with atrial septal defect. Med Arch. 2013; 67(2):141-142.

[3] Samartzis D, Herman J, Lubicky JP, Shen FH. Sprengel's deformity in Klippel-Feil syndrome. Spine. 2007; 32(18): E512-E516.

[4] Schreiber C, Bleiziffer S, Kostolny M, Hörer J, Eicken A, et al, Minimally invasive midaxillary muscle sparing thoracotomy for atrial septal defect closure in pre-pubescent patients. Ann Thorac Surg. 2005; 80(2):673-676.

[5] Madhurita S, Rupam P, Rebecea J. Anaesthetic challenges in a patient with Klippel-Feil syndrome undergoing surgery. Indian J Anaesth. 2005; 49(6):511-514. 